\title{
Pain Flashbacks in Posttraumatic Stress Disorder
}

\author{
Timothy V. Salomons, BA, BSc, * Janet E. Osterman, $M D, \uparrow$ Lucia Gagliese, $P h D, \S^{*} \ddagger$ and \\ Joel Katz, PhD\$千ף*
}

\begin{abstract}
Objectives: Surgical patients who regain consciousness while under general anesthesia may develop symptoms of Posttraumatic Stress Disorder (PTSD). One common PTSD symptom is the experiencing of abnormal perceptions during which the patient feels as if the trauma is recurring. The objective of this report is to document the re-occurrence of pain as part of the PTSD sequelae.
\end{abstract}

Results: We present two patients who developed PTSD following an episode of awareness under anesthesia. In both cases, posttraumatic sequelae persisted for years and included pain symptoms that resembled, in quality and location, pain experienced during surgery. In addition to their similarity to the original pain, these pain symptoms were triggered by stimuli associated with the traumatic situation, suggesting that they were flashbacks to the episode of awareness under anesthesia.

Discussion: The similarity between the patients' pain symptoms and pain experienced during trauma, the triggering by traumatic cues, and the associated emotional arousal and avoidance suggest the involvement of a somatosensory memory mechanism.

Key Words: posttraumatic stress disorder, pain memory, somatosensory memory, surgery, awareness under anesthesia, flashbacks

urgical patients who regain consciousness while under $\checkmark$ general anesthesia frequently find themselves powerless to alert the anesthesiologist or surgeon to their plight, due to the use of muscle relaxants. ${ }^{1-4}$ These patients may subsequently develop PTSD, ${ }^{5}$ a psychiatric condition characterized by symptoms of re-experiencing, avoidance, emotional numbing, and hyperarousal. ${ }^{6}$ In a recent review of the link between

From the *Department of Anesthesia and Pain Management, University Health Network and Mount Sinai Hospital, Toronto, Ontario, Canada; †Department of Psychiatry, Boston University School of Medicine, Boston, Massachusetts; qDepartment of Psychology, $\$$ School of Kinesiology and Health Science, York University, Toronto, Ontario, Canada; and §Department of Anesthesia, University of Toronto, Ontario, Canada.

Reprints: Joel Katz, PhD, Department of Anesthesia and Pain Management, University Health Network, EN 3-440, 200 Elizabeth Street, Toronto, Ontario, Canada, M5G 2C4 (e-mail: jkatz@uhnres.utoronto.ca). awareness under anesthesia and PTSD, Osterman and van der Kolk ${ }^{1}$ noted that inescapable stress is particularly conducive to the development of PTSD symptoms.

One characteristic symptom of PTSD is "acting or feeling as if the traumatic event were recurring." Patients with PTSD often report flashbacks, in which actual sensations related to their trauma are re-experienced. ${ }^{7}$ Mellman and Davis ${ }^{8}$ noted that visual flashbacks are most common but abnormal perceptions occur in other modalities, often triggered by heightened arousal or sensory cues. They report on a combat veteran who re-experienced "pain like a shrapnel wound" as part of combat-related posttraumatic sequelae, indicating that pain may occur as a sensory flashback to trauma.

The re-occurrence of pain as a sensory memory has been well documented in the amputee population, ${ }^{9}$ but, with a few exceptions, ${ }^{10}$ has been otherwise virtually ignored. The object of the present clinical note is to report two patients who became aware during surgery while under general anesthesia. The patients subsequently developed PTSD symptoms that included pain similar in quality and location to the pain they experienced when they awoke during surgery.

Consent was obtained from both patients to be interviewed and to have their history published. Both patients were interviewed using two standardized measures, the Clinician Administered PTSD Scale ${ }^{11}$ (CAPS) and the Traumatic Memory Inventory ${ }^{12}$ (TMI). The CAPS is a validated structured clinical interview, targeted to a specific event that meets diagnostic and statistical manual-IV (DSM-IV) stressor criterion (experiencing a threat to one's body integrity and responding with helplessness or terror). ${ }^{6}$ The CAPS assesses the frequency and intensity of 17 core, and 8 associated, symptoms of PTSD, as well as level of functional impairment. CAPS scores range from 0-136 with scores of 45 or above meeting DSM-IV diagnostic criteria with symptoms of moderate severity. The TMI is a 60 -item structured interview developed for the systematic collection of data concerning the retrieval of an emotionally significant traumatic memory. The memory is examined in three time periods: initially, when the memory was most distressing, and at the time of the interview. The interviews were conducted in the context of a study ${ }^{1}$ assessing posttraumatic sequelae following awareness under anesthesia. Documentation of pain flashbacks was not an a priori objec- 
tive of that study. It was during administration of the TMI that the pain memories presented here were first revealed by the patients.

\section{CASE HISTORIES}

\section{Case 1: Nocturnal Back Pain}

R.V., a 68-year-old retired woman, was interviewed 13 years after a laminectomy under general endotracheal anesthesia, as verified by review of her surgical record. Her anesthesia record revealed an uneventful surgical experience and did not indicate that she experienced awareness under anesthesia. (This discrepancy is consistent with research showing little correspondence between surgical records and self-report of awareness under anesthesia. ${ }^{3}$ )

R.V. reported that she awoke several times during her surgical procedure, experiencing an excruciating burning pain in her back and down her left leg. She was unable to move or speak and could not alert the anesthesiologist that she was awake. She reported feeling a sense of terror and an overwhelming desire to flee. She recalled hearing the surgical team discuss the S2 nerve, and she attributed the pain to movement of the S2 nerve. R.V. also recalled hearing clinking of instruments and scraping noises as well as "chit chat." She did not report this experience to the surgeon, anesthesiologist, or other hospital staff in the recovery room after surgery for fear of a negative reaction. She also did not confide in her family or any subsequent physician.

R.V. suffered from chronic severe back pain following the laminectomy which caused her to retire early. In addition to a disabling daytime back pain, she experienced a distinct nocturnal pain in her back and leg. This pain appeared to be triggered by a state of consciousness resembling a light anesthetic state. R.V. recounted that every night since the surgery, she would drift off into a light sleep, with the aid of a hypnotic medication, and suddenly be awakened by an intense, burning pain in her back and leg. This pain was different in quality and location from the dull pain she felt during the day, which was limited to her back. R.V. commented, "Isn't it surprising-it is the identical pain to the pain I had in the surgery!" R.V. also described feeling a strong urge to move, similar to her feeling during surgery, and was compelled to get out of bed and walk around the room. Exhausted, she would return to bed, only to re-experience the back and leg pain and desire to walk about, many times each night. Interestingly, R.V. also had vivid memories of the sound of the instruments "clinking and scraping" and for many years following her surgery could not eat with metal utensils.

R.V. became a high utilizer of medical services following her laminectomy, seeking relief from her severe nocturnal back and leg pain and sleep disorder. She received a disability pension for an unrelated cardiac condition. Over the years, she had received a variety of treatments for her sleep and pain com- plaints, including benzodiazepines, barbiturates, and multiple analgesic agents. She also underwent a second laminectomy with great reluctance and fear in the hope that her debilitating pain would be relieved. The procedure was unsuccessful.

At the time of the interview, R.V. had a CAPS score of 81 and a clinical diagnosis of PTSD. She received traumafocused cognitive-behavioral therapy (CBT) for her awareness-induced PTSD with resolution of her nocturnal reexperiencing of back and leg pain and improved sleep. Her chronic daytime back pain, due mostly to an arthritic condition, was unchanged.

\section{Case 2: Substernal Chest Pain}

P.K., a 43-year-old woman, who was employed in a hospital, suffered awareness under anesthesia during a laparoscopic tubal ligation, 3 years prior to the interview. Her medical record described an uncomplicated surgery under general endotracheal anesthesia with no indication of awareness under anesthesia. Her preoperative examination revealed a healthy woman with a normal EKG.

P.K. gave a detailed account of her surgical experience, indicating that she only briefly went under anesthesia. She described intubation and extubation and experienced significant pain and distress throughout the surgery. She described urgent efforts to move and communicate with her doctor, but was unable to "make a sound or even move a finger." She reported being able to open her eyes just enough to see details of the surgical environment but not enough to be detected. She attempted to elicit help but felt "defeated." P.K. was able to give exact details describing the procedure, which matched with the surgeon's report.

Despite multiple symptoms of PTSD, including flashbacks and nightmares of the laparoscopy, P.K. returned to work several weeks after surgery. At that time, she reported having been in an elevator with a nurse in a blue scrub suit and mask when she began experiencing severe substernal chest pain, which she described as a "painful pressure, pushing outward against my sternum." The pain was located from just below the sternal notch to the angle of Louis, coinciding with the location of the pain during intubation. P.K. described seeing the nurse and experiencing a sense of being trapped and of wanting to flee the elevator. She reported feeling disconnected from her surroundings and "unreal." She then began to experience the pressure pushing out against her sternum. As she left the elevator, the pain resolved. Since that time, she avoided elevators even if she needed to walk multiple flights of stairs. She also became adept at avoiding scrub suits, eating in the cafeteria and going to areas where people in scrub suits might be. Despite her efforts, P.K. reported that each time she witnessed a blue scrub suit in the hospital, the community, or even on television, she suffered from this identical chest pain and the sense of disconnect or unreality. In time, simply seeing the color blue was sufficient to trigger this chest pain. She attrib- 
uted the chest pain to reminders of her trauma, but on the advice of her therapist, sought a medical evaluation. Medical evaluation including a cardiac work-up was negative, ruling out cardiac abnormalities as a cause of the pain.

P.K. had a CAPS score of 75 and a clinical diagnosis of PTSD. She received individual trauma focused CBT with resolution of the PTSD. In follow-up, P.K. reported some residual chest pain when seeing scrub suits but this pain was milder in nature. She no longer met diagnostic criteria for PTSD.

\section{DISCUSSION}

The cases presented here indicate that the posttraumatic sequelae of PTSD may include the reoccurrence of pain first experienced during the trauma (awareness under anesthesia in the present cases). These pain flashbacks are not simply cognitive recollections of a past pain but are described as actual sensations that resemble, in quality and location, the pain experienced during the original event.

There is little documentation in the non-amputee population to support the idea that pain can re-occur as a memory. Lenz and colleagues found that the pain and emotional responses associated with both angina ${ }^{13}$ and atypical chest pain $^{14}$ were reliably evoked by thalamic microstimulation but only in patients who had previously experienced these pains. These reports suggest that electrical stimulation during surgery re-activated a central representation of the sensory and affective dimensions of previously experienced pains.

The unique aspect of the cases we report is that the pain flashbacks are triggered by stimuli associated with the trauma, indicating that conditioning may have occurred at, or near, the time of trauma. R.V.'s pain was triggered by a state of consciousness resembling an incomplete anesthetic state. P.K.'s pain occurred after witnessing blue scrub suits, a reminder of her surgical trauma. Consistent with a conditioning hypothesis, P.K. also demonstrated stimulus generalization, with blue objects other than scrub suits eventually eliciting the pain.

Taken together, these case reports suggest that fear conditioning is involved in the re-activation of the patients' pain. Fear conditioning is a well-characterized phenomenon in which a neutral stimulus (such as a tone) comes to evoke amygdala-mediated behavioral, autonomic, and endocrine fear responses through previous association with a naturally fearevoking stimulus (such as a shock). ${ }^{15}$ The hypothesis that fear conditioning is involved in the establishment of the pain symptoms documented here is plausible in light of evidence indicating that learning can occur under anesthesia ${ }^{16-18}$ and that the extent of learning is inversely related to the depth of anesthesia. $^{19}$

This interpretation is also consistent with the patients' PTSD symptoms. Research has shown that memories for traumatic events differ qualitatively from everyday memories, and are often reported as precise images, smells, and physical sensations rather than conscious, declarative recollections. ${ }^{12}$ Fear conditioning has been proposed as a mechanism for posttraumatic memory alterations, ${ }^{20}$ including flashbacks in other sensory modalities. $^{21}$

Fear conditioning typically results in emotional or behavioral responses. To the best of our knowledge, the possibility has not been examined that fear conditioning might also result in the perceived re-activation of a somatosensory event such as pain. To explain how this might occur, the effects of the conditioned response on sensory, emotional, and cognitive dimensions of pain perception will be briefly examined.

On a sensory level, conditioned fear may alter nociceptive thresholds through both peripheral and central mechanisms. Fear results in peripheral catecholamine release ${ }^{22}$ which is known to lower nociceptive thresholds. ${ }^{23}$ Central alteration of nociceptive thresholds could occur by way of a descending amygdala-periacquaductal gray (PAG)-rostral ventromedial medulla (RVM)-dorsal horn pathway which has been implicated in conditioned analgesia and antianalgesia. ${ }^{24.25}$ Both peripheral catecholamine release and activation of the above mentioned central circuit would result in global, rather than topographically selective modulation of pain (Fields, personal communication, April 4, 2002). These processes could, however, contribute to sub-threshold nociceptive activity (resulting from lingering damage or sensitization sustained during trauma) being raised to threshold and experienced as a recurrence of pain.

On an emotional level, the conditioned fear response could add to the overall experience of pain by increasing the unpleasantness of the experience. The conditioned fear response is commonly experienced as unpleasant, negative affect. Negative affect (similar to that evoked by fearconditioned cues) is an integral part of the pain experience. ${ }^{26}$ Thus, an evoked fear response may not only raise nociception to supra-threshold levels but may add to the aversiveness of that experience by increasing the emotional distress associated with the somatic sensation. In some cases, this could result in an otherwise innocuous somatic sensation in the previously affected area being perceived as painful and aversive.

On a cognitive level, the fear response could lead to the pain and accompanying affect being interpreted in relation to the original trauma. The fear elicited by the conditioned stimulus would not only resemble the original emotional response to trauma but could be accompanied by memory fragments associated with the traumatic event, including flashbacks in other sensory modalities. While the fear response itself is not under conscious control, the similarity of the affective responses and the association with traumatic memory fragments could lead to the interpretation that the pain is being re-experienced.

There are limitations to these case reports. Both patients were interviewed years after the episode of awareness under anesthesia. Thus, the interpretation that their pain was the same as that experienced during the episode of awareness under anesthesia is retrospective and vulnerable to the biases inherent 
in recall of pain. ${ }^{27}$ This is a common limitation in studies of traumatic memory. Other traumatized populations, including holocaust survivors, ${ }^{28}$ war veterans, ${ }^{29}$ and survivors of child abuse, ${ }^{30}$ are routinely assessed for memory and psychiatric symptoms many years after the target event. Prospective documentation of the original and subsequent pain symptoms using standardized techniques would be helpful in determining the extent to which the re-experienced pain resembles the original pain.

The case of P.K. illustrates overlap between symptoms associated with panic attack and posttraumatic flashbacks. ${ }^{8} \mathrm{An}$ alternate account of her symptoms is that she was experiencing the chest pain commonly associated with panic attacks. The localization of chest pain to a small circumscribed area coinciding with the location of the endotracheal tube, however, makes this interpretation unlikely.

Finally, interpreting clinical symptoms in terms of fear conditioning is potentially problematic since the relationship between stimulus and response may be complicated by factors that cannot easily be studied outside a well controlled laboratory. For instance, it is difficult to explain why P.K.'s symptoms were provoked by blue scrub suits rather than other features of the surgical environment, or why R.V. did not experience pain when she heard "chit chat" or the clinking of utensils. In assessing the role of learning in the acquisition and maintenance of PTSD symptoms, Foa et $\mathrm{al}^{31}$ point out that factors such as cognitive appraisal, intensity and predictability of the trauma, and processes such as generalization, avoidance conditioning, and higher-order conditioning all complicate clinical presentation. An associative network theory such as that proposed by Foa and Kozak ${ }^{32}$ is less parsimonious than a simple fear conditioning explanation, but may account for the more complex forms of learning that occur during traumatic events.

The difficulty in unambigously interpreting these symptoms as pain flashbacks indicates that better documentation and research are needed. It is important that cases like these are presented, however, since this phenomenon has both clinical and theoretical significance. While incidence and prevalence statistics are unavailable, clinicians frequently refer to somatic flashbacks without a body of research or diagnostic categories to support their interpretations. Furthermore, there is a large body of literature documenting a host of poorly understood somatic symptoms in traumatized patients, ${ }^{33,34}$ indicating that current etiological explanations for pain are not providing answers for a significant portion of this population both in terms of explaining the origins of the pain and planning effective treatments.

Further documentation of cases such as these might elucidate the link between unexplained pain symptoms and psychological trauma by clarifying the role of well-characterized phenomena such as fear-conditioning. The etiological link between fear-inducing traumatic situations and intrusive memory symptoms is well documented..$^{35,36}$ The complex relationship between fear and chronic pain ${ }^{37}$ has been recently outlined, but the relationships among extreme fear, memory, and pain perception have yet to be studied. Information on how these processes interact may bring us closer to understanding the relationship between psychologic trauma and poorly explained somatic symptoms.

\section{CONCLUSIONS}

We present two cases of pain flashbacks that persisted for years after the patients suffered a traumatic episode of awareness under anesthesia. These flashbacks resembled the pain experienced during the episode of surgical awareness and with time were reliably triggered by specific environmental or internal stimuli, suggesting a classically conditioned response. The similarity in quality and location of the flashbacks to the original pain experience, the triggering of flashbacks by trauma-related cues, and the associated emotional arousal and avoidance suggest the involvement of a somatosensory memory mechanism. Research is needed to determine the incidence of pain flashbacks after traumatic injury and the mechanisms responsible for their establishment and maintenance.

\section{ACKNOWLEDGMENTS}

The authors would like to acknowledge Dr. Peter Stenn for the clinical insight that initiated this project. Supported in part by grant MT12052 from the Canadian Institutes of Health Research (CIHR), Ottawa, Ontario, Canada, and a CIHR Investigator Award to Joel Katz.

\section{REFERENCES}

1. Osterman JE, van der Kolk BA. Awareness during anesthesia and posttraumatic stress disorder. Gen Hosp Psychiatry. 1998;20:274-281.

2. Kiviniemi $\mathrm{K}$. Conscious awareness and memory during general anesthesia. AANA Journal. 1994;62:441-449.

3. Moerman N, Bonke B, Oosting J. Awareness and recall during general anesthesia: facts and feelings. Anesthesiology. 1993;79:454-464.

4. Blacher RS. On awakening paralyzed during surgery: a syndrome of traumatic neurosis. JAMA. 1975;234:67-68.

5. Osterman JE, Hopper J, Heran WJ, et al. Awareness under anesthesia and the development of posttraumatic stress disorder. Gen Hosp Psychiatry. 2001;23:198-204.

6. American Psychiatric Association. Diagnostic and Statistical Manual of Mental Disorders. 4th ed. Washington, DC: American Psychiatric Association; 1994.

7. Sierra M, Berrios GE. Flashbulb memories and other repetitive images: a psychiatric perspective. Compr Psychiatry. 1999;40:115-125.

8. Mellman TA, Davis GC. Combat-related flashbacks in postraumatic stress disorder: phenomenology and similarity to panic attacks. J Clin Psychiatry. 1985;46:379-382.

9. Katz J, Melzack R. Pain "memories" in phantom limbs: review and clinical observations. Pain. 1990;43:319-336.

10. Schreiber S, Galai-Gat T. Uncontrolled pain following physical injury as the core-trauma in post-traumatic stress disorder. Pain. 1993;54:107-110.

11. Blake DD, Weathers FW, Nagy LM, et al. The development of a clinicianadministered PTSD scale. J Trauma Stress. 1995;8:75-90.

12. van der Kolk BA, Fisler R. Dissociation and the fragmentary nature of 
traumatic memories: overview and exploratory study. J Trauma Stress. 1995;8:505-525.

13. Lenz FA, Gracely RH, Hope EJ, et al. The sensation of angina can be evoked by stimulation of the human thalamus. Pain. 1994;59:119-125.

14. Lenz FA, Gracely RH, Romanoski AJ, et al. Stimulation in the human somatosensory thalamus can reproduce both the affective and sensory dimensions of previously experienced pain. Nat Med. 1995;1:910-913.

15. LeDoux JE. Emotion circuits in the brain. Annu Rev Neurosci. 2000;23: 155-184.

16. Ghoneim MM, Block RI. Learning and consciousness during general anesthesia. Anesthesiology. 1992;76:279-305.

17. Adams DC, Hilton HJ, Madigan JD, et al. Evidence for unconscious memory processing during elective cardiac surgery. Circulation. 1998; 98:II289-92; discussion II292-3.

18. Munte S, Kobbe I, Demertzis A, et al. Increased reading speed for stories presented during general anesthesia. Anesthesiology, 1999;90:662-669.

19. Lubke GH, Kerssens $\mathrm{C}$, Phaf $\mathrm{H}$, et al. Dependence of explicit and implicit memory on hypnotic state in trauma patients. Anesthesiology. 1999;90: 670-680.

20. Shalev AY, Ragel-Fuchs Y, Pitman RK. Conditioned fear and psychological trauma. Biol Psychiatry. 1992;31:863-865.

21. Charney DS, Deutch AY, Krystal JH, et al. Psychobiologic mechanisms of posttraumatic stress disorder. Arch Gen Psychiatry. 1993;50:295-305.

22. Yehuda R. Biology of posttraumatic stress disorder. J Clin Psychiatry. 2000;61:14-21

23. Devor M, Seltzer Z. Pathophysiology of damaged nerves in relation to chronic pain. In: Wall PD, Melzack R, eds. Textbook of Pain. 4th ed. Edinburgh: Churchill Livingstone; 1999:129-164.

24. Fields $\mathrm{H}$. Pain modulation: expectation, opioid analgesia and virtual pain. Prog Brain Res. 2000;122:245-253.

25. Fields H, Basbaum A. Central nervous system mechanisms of pain modu- lation. In Wall PD, Melzack R, eds. Textbook of Pain. 4th ed. Edinburgh: Churchill Livingstone; 1999:309-329.

26. Chapman C. Limbic processes and the affective dimension of pain. Prog Brain Res. 1996;110:63-81.

27. Pincus T, Morley S. Cognitive-processing bias in chronic pain: a review and integration. Psychol Bull. 2001;127:599-617.

28. Yehuda R, Kahana B, Schmeidler J, et al. Impact of cumulative lifetime trauma and recent stress on current posttraumatic stress disorder symptoms in holocaust survivors. Am J Psychiatry. 1995;152:1815-1818.

29. Marmar CR, Weiss DS, Schlenger WE, et al. Peritraumatic dissociation and posttraumatic stress in male Vietnam theater veterans. Am JPsychiatry. 1994;151:902-907.

30. Briere J, Conte J. Self-reported amnesia for abuse in adults molested as children. J Trauma Stress. 1992;6:21-32.

31. Foa E, Steketee G, Rothbaum B. Behavioral/Cognitive conceptualizations of post-traumatic stress disorder. Behavior Therapy. 1989;20:155176.

32. Foa E, Kozack M. Emotional processing of fear: exposure to corrective information. Psychol Bull. 1986;99:20-35.

33. Andreski $\mathrm{P}$, Chilcoat $\mathrm{H}$, Breslau N. Post-traumatic stress disorder and somatization symptoms: a prospective study. Psychiatry Res. 1998;79: 131-138.

34. Asmundson GJ, Norton GR, Allerdings MD, et al. Posttraumatic stress disorder and work-related injury. J Anxiety Disord. 1998;12:57-69.

35. Roozendaal B, Quirarte GL, McGaugh JL. Stress-activated hormonal systems and the regulation of memory storage. Ann N Y Acad Sci. 1997;821: 247-258.

36. Cahill $\mathrm{L}$. The neurobiology of emotionally influenced memory: implications for understanding traumatic memory. Ann N Y Acad Sci. 1997;821: 238-246.

37. Turk $D$. A diathesis-stress model of chronic pain and disability following traumatic injury. Pain Res Manag. 2002;7:9-19. 